\title{
Numerical simulation of double jet impinging a flat and fixed wall with WALE model
}

\author{
Rachid Sahnoun ${ }^{1}$, Aissa Abderrahmane ${ }^{2}$, Drai Ismail ${ }^{1}$, Mohmmed El Ganaoui ${ }^{2}$ \\ ${ }^{1}$ Université de Mustapha Stambouli 29000-Mascara-Algérie; e-mail: r.sahnoun@univ-mascara.dz \\ ${ }^{2}$ Laboratoire de Physique Quantique de la Matière et Modélisation Mathématique (LPQ3M), Université Mustapha Stambouli de \\ Mascara, Algeria \\ ${ }^{3}$ Laboratoire énergétique de Longwy (FJV/LERMAB), Université de Lorraine, France.
}

\begin{abstract}
This work focuses on the study of the averaged velocity and the turbulent intensity of the double jet impingement is used to separate two adjacent cellule. Numerical LES investigations are carried out in this study. A jet impingement against a flat and smooth surface without recirculation was used for this. The WALE (Wall Adapting Local Eddy) structure models the sub grid-scale tensor. The jet opening ratio is H/e $=10$ and the Reynolds Number $\mathrm{Re}=2000$. Result comparisons to available experimental measurements are performed and good agreements are noticed.
\end{abstract}

\section{1 introduction}

The separation of two media by a single jet is often used to reduce heat and mass transfer. Several experimental and numerical investigations evoke the fact that the double jets allow a better separation compared to the simple jet, because the intensity of axial turbulence is much lower [1], [2], [5]. This paper is a numerical simulation using the LES approach in order to make a detailed study of the behaviour of the double jet, whether vertically or horizontally. The simulation results are compared with experimental data from GUPTA [1].

Hsu et al [6] used a laser assisted smoke flow visualization tool and particle image velocimetry (PIV) to analyze the flow behaviors and time averaged speed fields of the characteristic flow structures. They found the convective heat transfer was improved by increasing the velocity of the vortex ring and the size of the fluctuations. Singh [7] has numerically studied the fluid flow characteristics of a turbulent offset jet that impinges on a wavy wall surface. they concluded that For both the near-stagnation and self-similar regimes the flow is found to be relatively isotropic. Such comprehensive observations are necessary to understand better the transfer of heat from impinging synthetic jets. Ziyan et al. [8] experimentally studied the flow dynamics of sweeping jets impinging upon confined concave surfaces at different Reynolds numbers and surface curvatures by time-resolved particle image velocimetry (TR-PIV). They show that The flow dynamics associated with the wall of intermediate curvature $(\mathrm{R}=20 \mathrm{Dh})$ generally show transitional behaviors

\section{The Basic Equations and the solution method}

\subsection{LES modelling}

The isothermal air jet flow is governed by the conservation equations of mass and momentum.

$$
\begin{gathered}
\frac{\partial \bar{u}_{i}}{\partial x_{i}}=0 \\
\frac{\partial \bar{u}_{i}}{\partial t}=\frac{\partial\left(\bar{u}_{i} \bar{u}_{j}\right)}{\partial x_{i}}=-\frac{1}{\rho} \frac{\partial \bar{P}_{i}}{\partial x_{i}}-\frac{\partial t}{\partial x_{j}}+v \frac{\partial^{2} \bar{u}_{i}}{\partial x_{i} \partial x_{j}} \\
\tau_{i j}=v_{S G S}\left(\frac{\partial \bar{u}_{i}}{\partial x_{j}}+\frac{\partial \bar{u}_{j}}{\partial x_{i}}\right)
\end{gathered}
$$

We can specify the eddy viscosity or the sub grid viscosity with the WALE model as:

$$
v_{S G S}=\left(C_{w} \Delta\right)^{2} \frac{\left(S_{i j}^{d} S_{i j}^{d}\right)^{\frac{3}{2}}}{\left(S^{d}{ }_{i j} S^{d}{ }_{i j}\right)^{\frac{5}{2}}+\left(S^{d}{ }_{i j} S^{d}{ }_{i j}\right)^{\frac{5}{4}}}
$$

\footnotetext{
* Corresponding author: author@e-mail.org
} 
where

$$
S^{d}{ }_{i j}=\frac{1}{2}\left(g_{i j}^{2}+g_{j i}^{2}\right)-\delta_{i j} g_{k k}^{2}
$$

and the constant $\mathrm{C}_{\mathrm{w}}=0.325$

$$
g_{i j}=\frac{\partial \bar{x}_{i}}{\partial x_{j}}
$$

\subsection{Computational domain and boundary conditions}

The two-jet configuration drawn from the experimental study of (Gupta 2005) [1] is showed in Fig. (2.1) and Fig. (2.2). The width $G$ of the calculation range is fixed at $1 \mathrm{~m}$, the height $\mathrm{H}$ at $0.3 \mathrm{~m}$ and the ratio $\mathrm{H} / \mathrm{e}$. represents the width of the blowing slot) is equal to 10 . The separation plate located at 1 inside the slot of the diffuser is $1 \mathrm{~mm}$ thick. The length of the geometry is $6 \mathrm{~m}$. The contraction ratio is $\mathrm{H} / \mathrm{e}=10$, the initial width of the blowing slot $\mathrm{e}=0.3 \mathrm{~m}$ is separated in two by a flat plate. The welded slot plate inside the blowing slot has a straight trailing edge generating a wake at the outlet of the blowing slot. Thus, the curtain with two jets is the result of two interactions in close jets with a wake, which are two simple streams sheared with free turbulence (apart from the confinement achieved by the ceiling and the impact wall). The proximity of the two jets generates lateral mixing layers preventing a free development of turbulence at the interface of the jets. The initial speed profile is $\mathrm{V}(0)=1 \mathrm{~m} / \mathrm{s}$ and concerning the Reynolds number is $\operatorname{Re}=2000$.

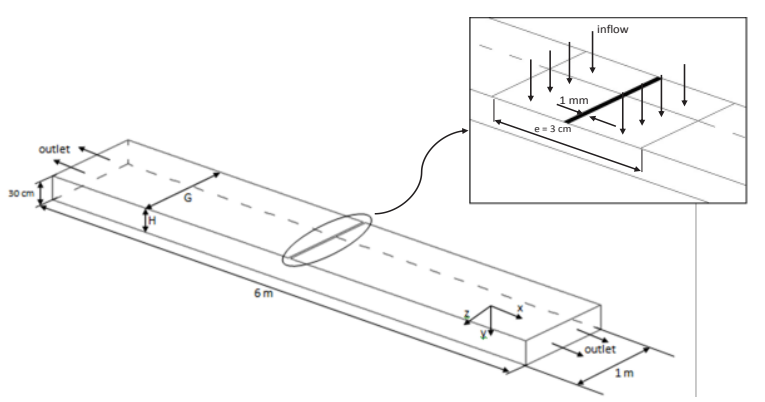

Fig. 2.1 The geometry of twin jet.

The numerical simulation was carried out under the environment of the ANSYS Fluent 15.0 calculation code. The fluid is assumed to be incompressible and Newtonian. The algorithm used is SIMPLE to solve the coupling between pressure and speed. This algorithm developed by (Patankar 1980) [4] is a correction procedure for calculating the pressure after the discretization of the Navier-Stokes equations. Spatial and temporal discretization will be integrated into an implicit second order scheme. The WALE model described by (Nicoud \& Ducros 1999) [3] is used for the modeling of the sub-mesh tensions via the turbulent viscosity.

A structured mesh of 700,000 cells is used for the simulation in $3 \mathrm{D}$ of the computation domain. A process of refining the grids is adopted around the vertical axis of the calculation domain and near the walls, Fig. (2.3). The no-slip condition is applied to the wall.

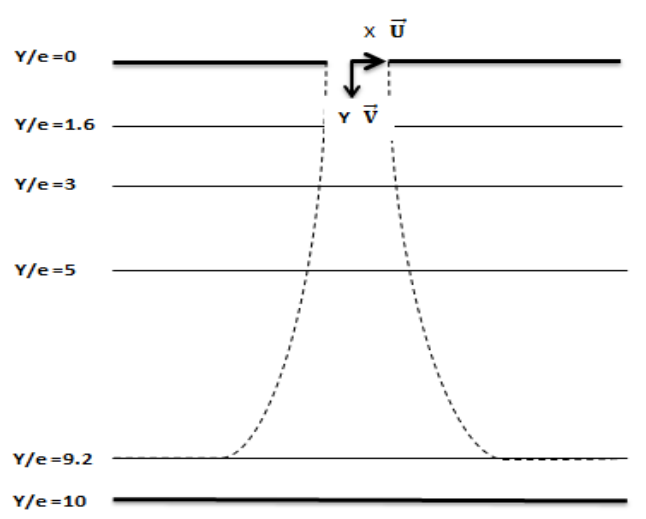

Fig. 2.2 the horizontals axes of interest.

All the results of the curtain configurations studied are compared with the experimental data provided by (Gupta 2005) for $\mathrm{Re}=2000$. The results will be presented along the vertical axis (oy) and at different horizontal stations along the axis (ox). Figure (2.2).

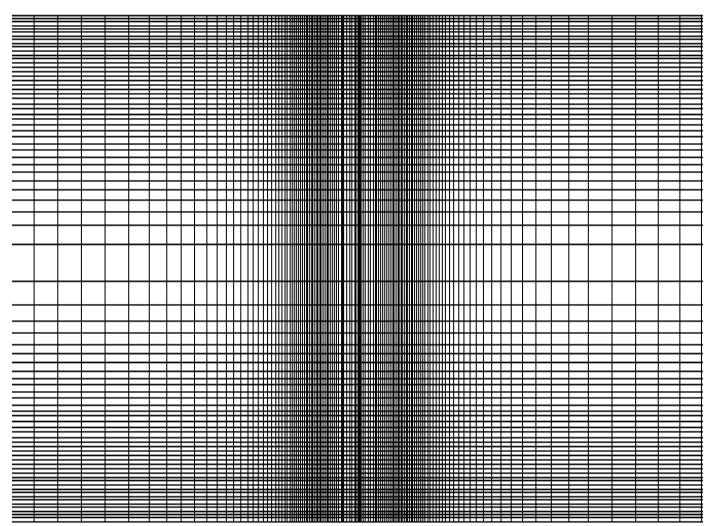

Fig. 2.3 Structured mesh.

\section{RESULTS AND DISCUSSION}

The Reynolds number is defined as $\operatorname{Re}=\frac{U_{0} v}{v}$ (where $\mathrm{U}_{0}$ is the maximal velocity air flow at the inlet jet and $\vartheta$ is the kinematic viscosity) is fixed to 2000 . The code was used to numerically solve the governing equations with segregated scheme and the SIMPLE algorithm solved the pressure-velocity coupling. This algorithm was originally put forward by Patankar and Spalding [15] and is essentially a guess and correct procedure for the calculation of pressure on the staggered grid arrangement. The spatial discretization and time integration were performed by a second-order implicit scheme. The WALE model described by Nicoud and 
Ducros [16] is used to model the SGS eddy viscosity until overcome the simulation problem at walls. Refined and structured mesh is used at near walls and at the vertical jet axe with 700000 cells. All results of the double jet SIM configurations are compared to the experimental data provided by Gupta [1] twin jets with $\mathrm{Re}=2000$. The results from the comparison of the five different jets application will be presented and discussed in four different horizontal direction locations (figure $(2.2)$ ).
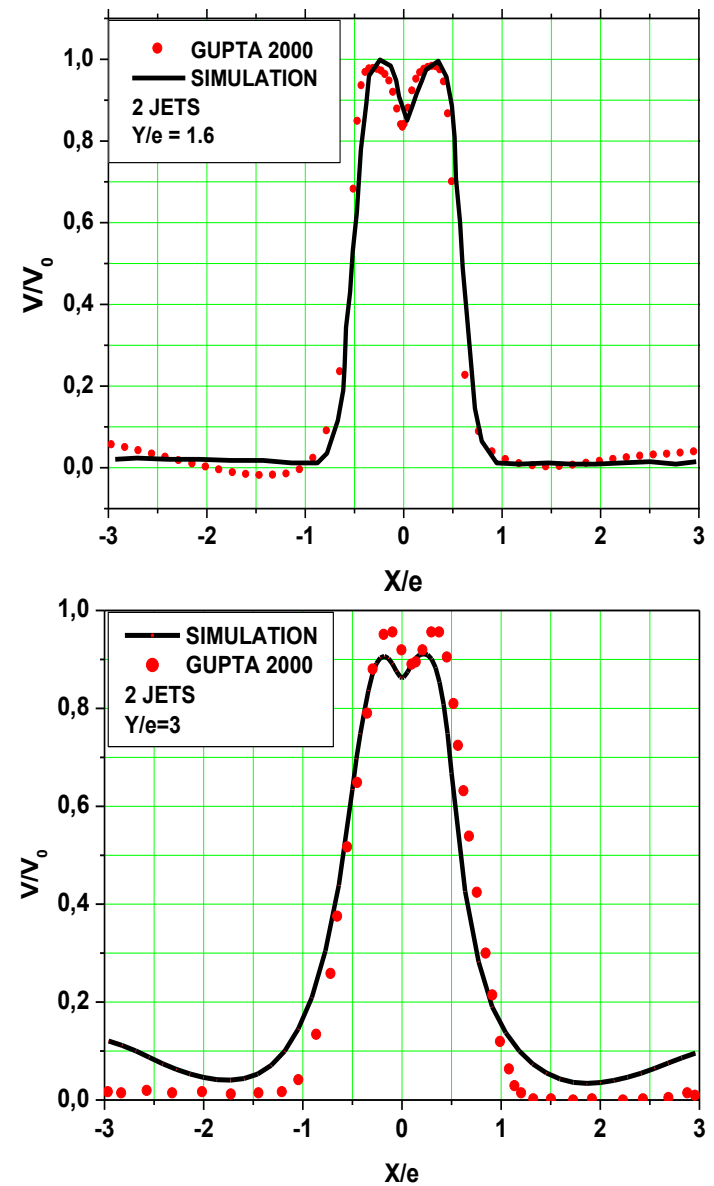
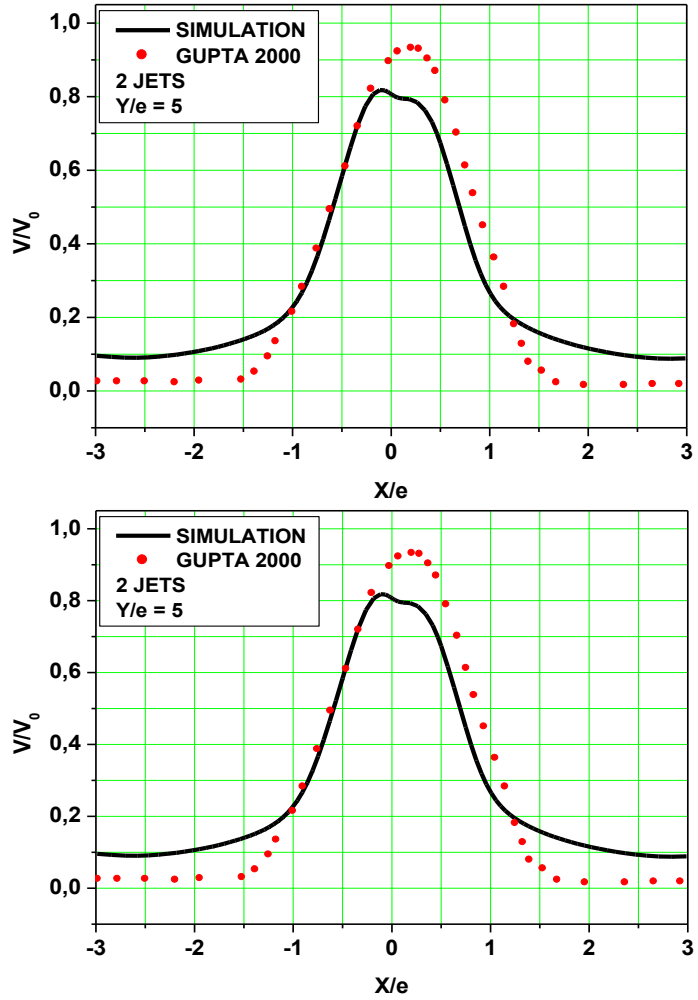

Fig. 3.1 The mean velocity in the $\mathrm{x}$ direction for the four stations.

La Figure (3.1) illustrates the profile of the velocity component $\mathrm{V} \rightarrow$ of the double jet at each of the four stations defined in Fig. (2.2). It emerges from the experimental data that the development of the lateral mixing layers of the two jets takes place beyond the station $\mathrm{y} / \mathrm{e}=3$. In addition, it appears once the mixing is established, that the peak of the speed profile is deviated from the vertical axis due to the asymmetry caused by the weld effect of the partition plate. We can say that the model is in good agreement with the experimental measurements up to the station $\mathrm{y} / \mathrm{e}=3$, then it begin to diverge because it does not take into account the mixing forces which are difficult to characterize. This type of force is still the subject of research work requiring substantial resources.

The Fig. (3.3) illustrates the speed profiles $U$ for the different stations selected. There is good agreement between the experimental data and the simulation results for the station $\mathrm{y} / \mathrm{e}=9.2$ in the interval $-1<\mathrm{x} / \mathrm{e}<1$. For the other stations this interval represents a refined mesh area which explains concordance of results. 

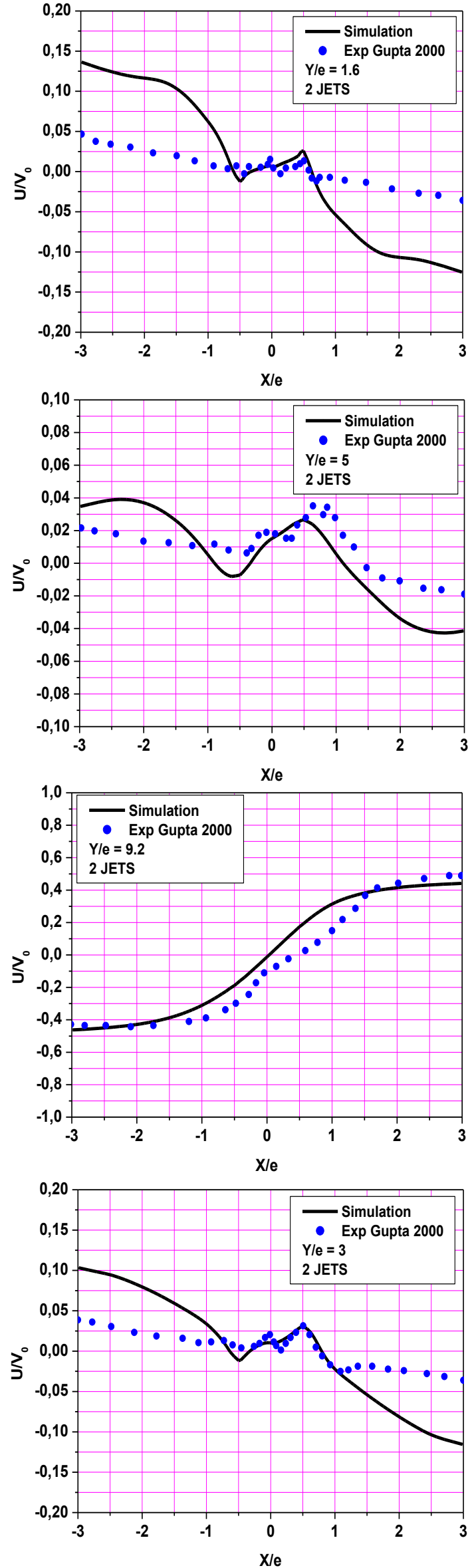

Fig.3.3 the normalized mean velocity $U$ in the $x$ direction for the all four stations.
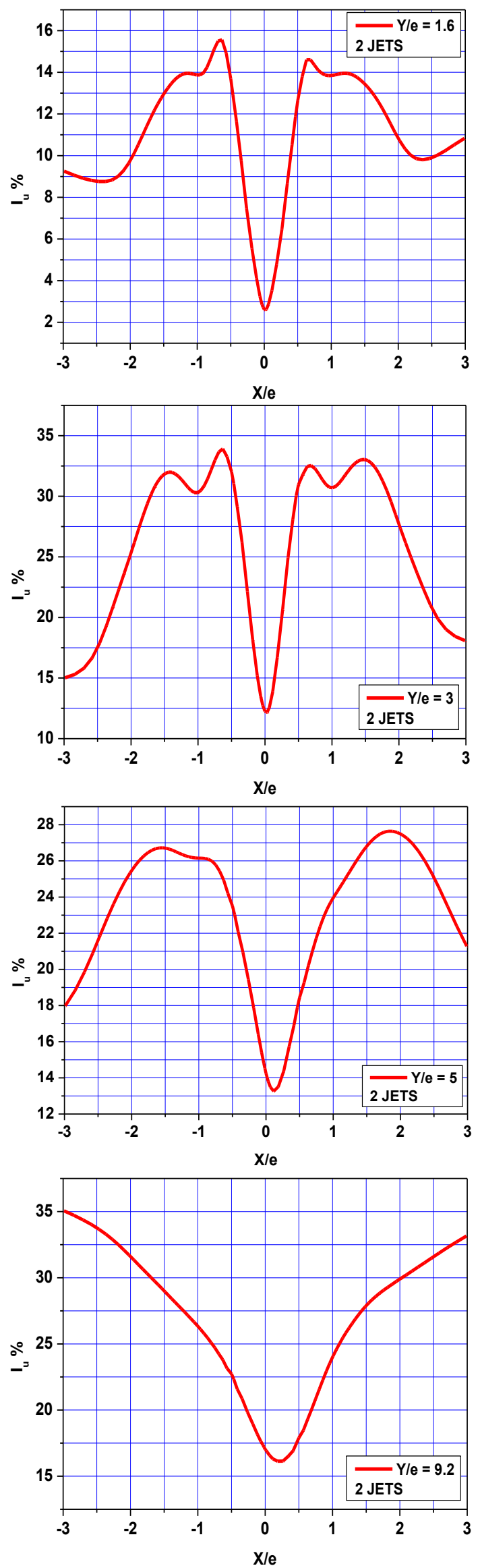

Fig. 3.4 Turbulent intensity in the y direction for the four stations. 

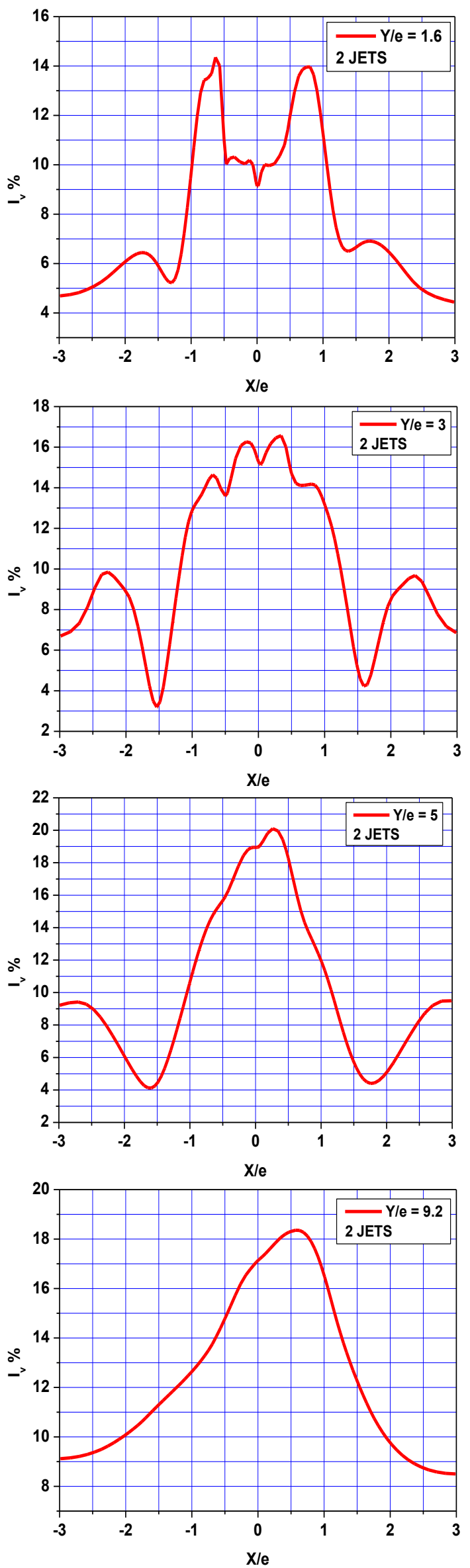

Fig. 3.5 Turbulent intensity in the $\mathrm{x}$ direction for the four stations.
Fig. (3.5) shows the profiles of the turbulent intensity I_(v )corresponding to the four selected stations. We observe for station $\mathrm{y} / \mathrm{e}=1.6$ two peaks on either side of the axis of the double jet while for the other stations the appearance of a single peak in the middle of the jet seems to be the result of the layers of mixed.

\section{Conclusion}

The numerical simulation of the double jet with LES approach and WALE model was treated in this paper. The results of this simulation show good coherence between experimental data and simulated ones especially near the vertical axe and at the impingement wall. All differences is due to the no refinement mesh far the vertical axe and due to the profile of the velocity and turbulent intensity profile at the inlet where in this simulation is uniform.

\section{References}

1. S. Gupta, PhD Thesis, Ecole des mines de NantesFrance (2005)

2. R. Sahnoun et al. Int. Rev. Mech. Eng. (IREME). 3, (2019).

3. F. Nicoud, F. Durcos, Kluwer Academic Pub. 62, 183 (1999).

4. S.V. Pantakar, Taylor \& Françis Group, New York. (1980).

5. N. Riviere, PhD Thesis, 3575. L'Université de Bordeaux I, France, (2008).

6. C.M. Hsu, W.C. Jhan, Y.Y. Chang, Heat Mass Transf. 56, 143 (2020).

7. T. Singh, A. Kumar and A. Kumar Satapathy, J. Mech. Eng. Sci., 234, (2020).

8. L. Ziyan, L. Jiajun, Z. Wenwu, L. Yingzheng, W. Xin, Int. J. Heat Mass Transf, 142, 118457 (2019). 